\title{
Dedicated WhatsApp Messenger Use in Neurosurgery: A Systematic Review
}

\author{
Taiwo Akhigbe ${ }^{1 *}$, Ardalan Zolnourian ${ }^{1}$ \\ ${ }^{1}$ Department of Neurosurgery, University Hospital Southampton, Southampton, UK
}

Corresponding Author: Taiwo Akhigbe, MBBS, MCh, MSc, PgD, Department of Neurosurgery, University Hospital Southampton, Tremona Rd, Southampton SO16 6YD, UK. E-mail: akhigbetaiwo@yahoo.com

Received April 22, 2017; Accepted June 3, 2017; Online Published June 29, 2017

\begin{abstract}
Introduction: Outdated communication technology in neurosurgery can place effective patient care and safety in jeopardy. Patients care in neurosurgery has significantly improved with the introduction of modern equipment and recent technology for effective and efficient patient care. WhatsApp represents as a safe, efficient, easily affordable and cost effective technology. This study aims to review the use of dedicated WhatsApp for facilitating communication in neurosurgery setting for the first time and discusses its attendant effect on ethics, professional and social implication.

Methods: A systematic literature search was performed to evaluate and analyse current evidence with regards to dedicated WhatsApp use in neurosurgery using databases including Medline, EMBASE and Google Scholar. The keywords employed in this search strategy include WhatsApp, social media, ethics, professionalism, and neurosurgery. The inclusion criteria are any type of study relevant to the review, studies on adult human patients only, papers only published in English and seminal papers relevant to this study.

Results: The systematic literature search yielded 750 articles. Furthermore, twelve studies were identified after the removal of nonrelevant studies and duplicates. Final screen of eligibility for dedicated WhatsApp Messenger usage yielded two articles. A prospective observational study by Kankane et al. and a letter by Graziano et al., which described in detail WhatsApp use in neurosurgical centers. Also, in five neurosurgical centers, dedicated WhatsApp use were identified. Currently there is significant paucity of evidence of WhatsApp use in Neurosurgery.

Conclusion: The inventor of WhatsApp has widely helped in developing a completely novel and innovative technology with the potential to improve patient care. This is while, the current form of WhatsApp has been considered to be unsafe in terms of ethical implication to handle patient data and eventually, is inappropriate for use in clinical environments. It can be mentioned that a more secure alternative will definitely come to use in clinical environments.

Keywords: WhatsApp; Social Media; Ethics; Professionalism; Neurosurgery

Citation: Akhigbe T, Zolnourian A. Dedicated WhatsApp messenger use in neurosurgery: a systematic review. Int J Med Rev. 2017;4(2):58-61. doi: 10.29252/ijmr-040206.
\end{abstract}

\section{Introduction}

Patients care in neurosurgery has significantly improved with the introduction of modern equipment and recent technology. In spite of all these developments, there are still many hospitals which use outdated pager systems for clinical communication between physicians and other health care professionals. Unfortunately, it has been obviously proven that the pager systems have been overflown with many problems such as high costs, long wait times for the return of a page, frequent interruptions, and also not being able to identify the location or identity of the caller which as a result places patient safety at a risk1. WhatsApp use is now commonplace among healthcare professionals of all sizes, levels and grades. It is said that the groups in
WhatsApp tend to create effective and simple communication among the team members. It allows discussions for both medical and administrative issues. It has been observed that without any formal setups within teams, WhatsApp groups appear to be used extensively. ${ }^{1}$ Going through the history of WhatsApp reveals that it was founded in 2009 and is globally approaching one billion users. It was at first acquired by Facebook in 2014 in a $\$ 19 B n$ acquisition. ${ }^{2}$ Since its outset, WhatsApp has experienced serious security concerns. At first, messages were sent over the Internet in simple text formats. Initial encryption attempts have been proved to be deficient and improper. ${ }^{3}$ With many social media platforms and developments within the 21 st century, the importance of

Copyright (C) 2017 The Author(s). This is an open-access article distributed under the terms of the Creative Commons Attribution License (http://creativecommons.org/licenses/by/4.0), which permits unrestricted use, distribution, and reproduction in any medium, provided the original work is properly cited. 
patient confidentiality and strict patient data protection has become much more significant.

On the 5th of April 2016, this application introduced an end-to-end encryption of all the messages sent on the mobile. This requires the user to have the latest version installed. This protocol actually prevents any party having $\mathrm{p}$ access to messages. As a matter of fact, it ensures that neither WhatsApp themselves nor any other third party can read any messages sent by a user. The only person who has direct access to the message is the intended receiver. This is due to the fact that only the recipient and sender have the required key to unlock and read the message. This is true about all message types including: chats, group chats, images, videos, voice messages, and files. Without any doubt, this fact is particularly important when considering group chats, which are widely used in medical teams. ${ }^{4}$ The unpredictable nature of working in neurosurgery departments either as residents or as consultant neurosurgeons who must be always available to review and manage extremely sick patients alongside performing emergency surgeries in addition with running clinics places a significant premium and priority on safe, clear and efficient communications. If the team is willing to function well and also intends to avoid clinical incidents, rapid and clear communications must be available all day long.

To this date, no review study has been conducted regarding the dedicated use of WhatsApp in neurosurgery. This study aims to review the dedicated WhatsApp Messenger usage for facilitating communication in neurosurgery and its ethics, its professional and also social implication.

\section{Methods}

A comprehensive literature search was performed to evaluate and analyse the evidence with regards to the dedicated WhatsApp use in neurosurgery using databases including Medline and EMBASE. Keywords employed in this search strategy included WhatsApp, social media, ethics, professionalism, and neurosurgery. The inclusion criteria included any type of study relevant to the review, studies on adult human patients, papers published in English, and well researched correspondence relevant to this study. Editorials and comments were excluded. There is lack of evidence and articles on this subject. In addition, extensive research has been carried out on neurosurgical institutions that have adopted dedicated WhatsApp Messenger usage.

\section{Results}

The systematic literature search yielded 750 articles. Furthermore, after removing the non-relevant and duplicates studies twelve studies were identified. Finally, two articles a prospective observational study by Kankane et al. ${ }^{5}$, and a letter by Graziano et al. ${ }^{6}$, which had described the use of WhatsApp in neurosurgical centres in detail were yielded (Figure 1).

In addition, five neurosurgical centres have been seen where dedicated WhatsApp use were identified (Table 1). Currently there is significant paucity of evidence of WhatsApp use in Neurosurgery.

\section{Articles identified through databases search}

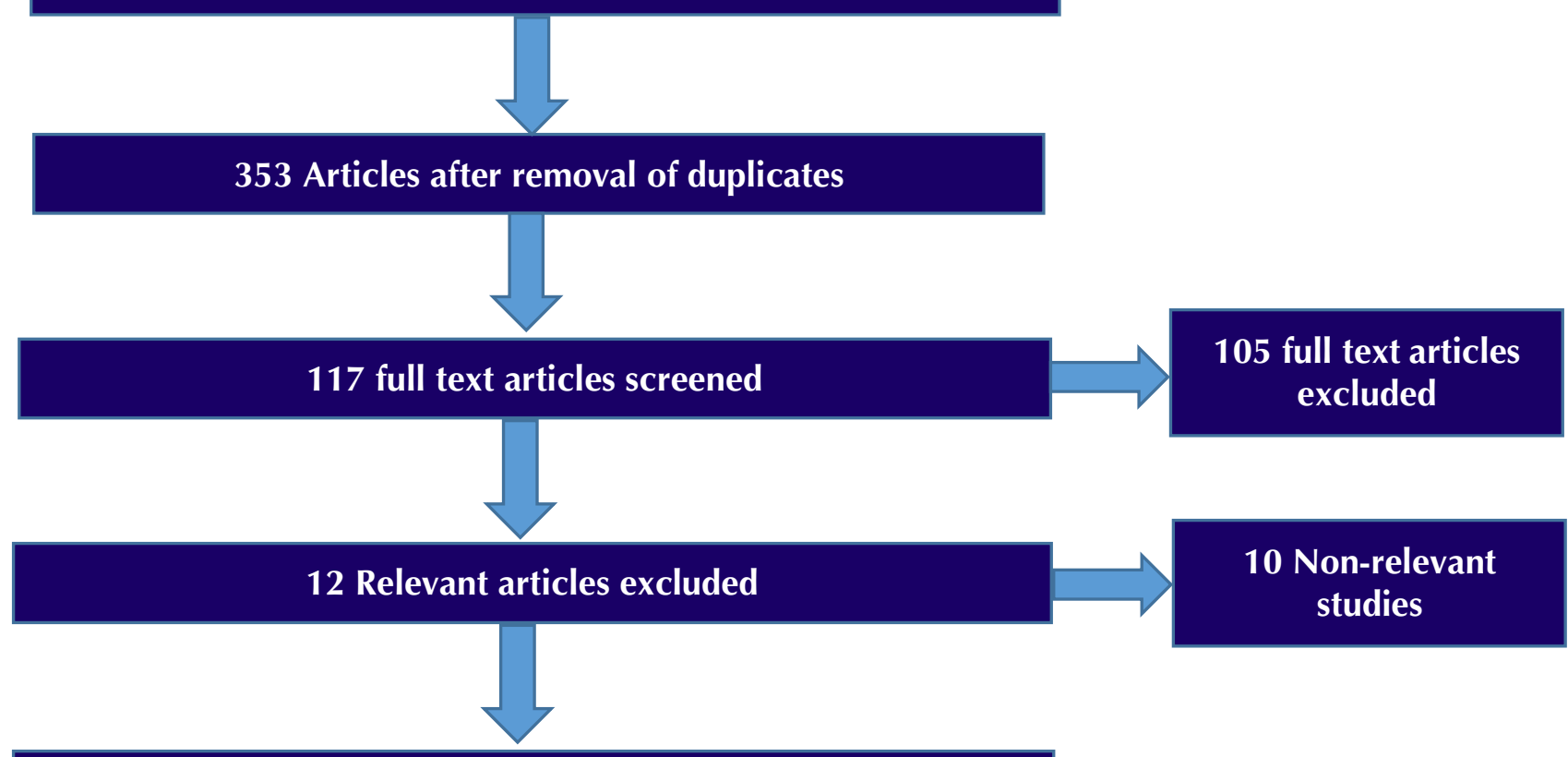

\section{Studies for systematic Review}

Figure 1. Search flow diagram 
Table 1. Neurosurgical centre where dedicated What App messenger is in use.

Location

RNT Medical college \& MB Hospital, Udaipur

Bey-Om-Sin, Brain Cord Nerve, Istanbul, www.bey-om-sin.com

Turkey

India

\section{Discussion}

In recent years, smart phones have become an essential tool for physicians in patient treatment, monitoring, rehabilitation and discussion of care strategies especially in emergency situations and where distance is an issue. Nowadays, it is usually more comfortable to view the computed tomography and magnetic resonance images, reports, and patients' neurological status immediately by using this App. Also, physicians can save time on making decisions and preparations regarding emergency surgery. Furthermore, special cases can be shared, and the opinions of different neurosurgeons can be gathered in the same group, for hopefully better treatments. Kankane et al. ${ }^{5}$ carried out a prospective observational study entitled "Apply of WhatsApp: A quick, simple, smarty and cost competent method of communication in Neurosurgery from December 2015 to June 2015". The results of this research is quite impressive despite the limitations of the study. 1,356 patients recruited, images received on WhatsApp took an average timing of 4.06 minutes, conflicts recorded in $7.22 \%$ cases between resident report and images on WhatsApp and all radiological imaging modalities were associated with statistical insignificant indifference. It was easy and fast enabling quick decision consequently early diagnosis and prompt treatment and above all cost effective. In another study, Graziano et al. ${ }^{6}$ attested to the dedicated WhatsApp use in their neurological centre in Italy. This centre used to share patient information, digital images and clinical suggestions with three chart groups of $\mathrm{NCH}$ (Neurosurgery) Palermo (Young and senior consultants), NCH Fellows (clinical and research fellows, student researchers) and $\mathrm{NCH}$ Palermo and Neuro Rad Messina (Neurosurgeons and Neuroradiologists). Ethical issues about regarding data protection and identity safety were not addressed by these two articles. In spite of its impressive results, ethics bordering dedicated use of WhatsApp in patient's care is a big issue with regards to effective and efficient patient confidentiality and adequate data security. The online world is characterized by a chronic lack of security. WhatsApp has decided to welcome development, however further studies is required to ascertain safe use in clinical settings.

During 2014, the National Health Service (NHS) advised not to use instant messaging in clinical information exchange due to its lack of relevant data security certification. ${ }^{7,8}$ The NHS code of practice emphasizes

\section{References}

1. Johnston MJ, King D, Arora S, Behar N, Athanasiou T, Sevdalis N, et al. Smartphones let surgeons know WhatsApp: this issue that all communications must comply with the 1998 Data Protection Act. Actually, WhatsApp disobeys this, and has violated both the Dutch and Canadian copyright laws, and has also been accused for violating the international copyright law. ${ }^{9-11}$ In the United States, The federal Health Insurance Portability and Accountability Act of 1996 in America is responsible to preserve all confidential health care information. According to WhatsApp's current status, it is unlikely to meet the required standards to transfer clinical data throughout the United States as regards to the Health Insurance Portability and Accountability Act of 1996. It is clear that WhatsApp is a much more cost-effective, user-friendly and hassle-free solution compared to the SMS. One of its special features is the "group chat" which gives this opportunity to people to be in touch with each other and share images and videos, with up to 50 members in a group. Moreover, it gives this possibility to physicians to easily discuss cases much faster and has also made the multidisciplinary consultations more efficient and complete. Considering this App as an intradepartmental communication tool, in the coming future, WhatsApp may be regarded as a global interhospital communication system, may reduce telemedicine costs and extend worldwide network connections. To our knowledge, this is the first comprehensive review of literature for WhatsApp messenger usage in neurosurgery, limited by very few studies

\section{Conclusion}

The inventor of WhatsApp has widely helped in developing a completely novel and innovative technology with the potential to improve patient care. This is while, the current form of WhatsApp has been considered to be unsafe in terms of ethical implication to handle patient data and eventually, is inappropriate for use in clinical environments. It can be mentioned that a more secure alternative will definitely come to use in clinical environments.

Authors' Contributions

TA and AZ contributed equally to this study.

Conflict of Interest Disclosures

Author declares that he has no conflict of interest.

Ethical Approval

Not applicable.

Funding

None.

an analysis of communication in emergency surgical teams. Am J Surg. 2015;209(1):45-51. doi: 10.1016/j.amjsurg.2014. 
08.030. pmid: 25454952.

2. Correspondent, Facebook to Buy Messaging App WhatsApp for \$19bn, BBC News R.T.A.T. [updated 2017April 11, 2015]. Available from: http://www.bbc.co.uk/news/business $-26266689$.

3. D'oh! Basic Flaw in WhatsApp Could Allow Attackers to Decrypt Messages. PCWorld 2013 [updated 2018; cited September 13, 2016]. Available from: https://goo.gl/wxu c9G.

4. WhatsApp Encryption Overview; Privacy and Security is in our DNA 2016 [updated 2018September 13, 2016]. Available from: https://www.whatsapp.com/security.

5. Kankane VK, Jaiswal G, Gupta TK. Apply of WhatsApp: A quick, simple, smarty and cost competent method of communication in neurosurgery. Romanian Neurosurg. 2016;30(2):306-12. doi: 10.1515/romneu-2016-0049.
6. Graziano F, Maugeri R, Giugno A, lacopino D. WhatsAPP in neurosurgery: the best practice is in our hands. Acta Neurochirurgica. 2016;11(158):2173-4.

7. NHS England, Risk alert-using apps to share data, NHS Engl Inf Gov Bull. Leeds: NHS England: 2014.

8. NHS England, WhatsApp "to be encrypted," NHS Engl Inf. Gov. Bull. Leeds: NHS England: 2015.

9. Bradshaw T, Dembosky A. WhatsApp Accused of Breaking Privacy Rules, Financial Times 2013 [updated 2017September 13, 2016]. Available from: https://goo.gl/JxGH6c.

10. Laver N. Companies bare burden for data protection, LexisNexis UK Leg. News Anal 2013 [September 13, 2016] Available from: https://goo.gl/6EG9Tn.

11. Hutchinson S, Rutgers L, Eilander M. News Release; WhatsApp's violation of privacy law partly resolved after investigation by data protection authorities 2013 [September 13, 2016]. Available from: https://goo.gl/ndQ6bH. 